\title{
CDK5RAP1 targeting NF-кB signaling pathway in human malignant melanoma A375 cell apoptosis
}

\author{
JIKUI XIONG ${ }^{1}$, YAN WANG $^{2}$, YANLI GU $^{3}$, YADONG XUE $^{4}$, LIN DANG $^{1}$ and YUZHEN LI ${ }^{1}$ \\ ${ }^{1}$ Department of Dermatology, The Second Affiliated Hospital of Harbin Medical University, Harbin, Heilongjiang 150086; \\ ${ }^{2}$ Department of Pathology, Heilongjiang Provincial Hospital, Harbin, Heilongjiang 150036; ${ }^{3}$ Department of Dermatology, \\ Daqing Oilfield General Hospital, Daqing, Heilongjiang 163000; ${ }^{4}$ Department of Dermatology, The First Affiliated \\ Hospital of Harbin Medical University, Harbin, Heilongjiang 150001, P.R. China
}

Received June 7, 2016; Accepted November 20, 2017

DOI: $10.3892 / \mathrm{ol} .2018 .7920$

\begin{abstract}
Malignant melanoma is characterized by rapid deterioration, early metastasis and high mortality. Cdk5 regulatory subunit-associated protein 1 (CDK5RAP1), which catalyzes 2-methylthio $\left(\mathrm{ms}^{2}\right)$ modification of mitochondrial transfer RNAs, has been reported to induce cancer cell apoptosis, by a phospho-c-Jun N-terminal kinase (p-JNK) signaling pathway. The present study was the first to report on the association between CDK5RAP1 deficiency and nuclear factor- $\kappa \mathrm{B}$ $(\mathrm{NF}-\kappa \mathrm{B})$ signaling pathway during the apoptosis process in human malignant melanoma (A375) cells. CDK5RAP1 small interfering RNA (siRNA) and control siRNA were transfected into A375 cells. CDK5RAP1 deficiency inhibited $\mathrm{Ca}^{2+}$ influx in A375 cells. CDK5RAP1 deficiency also suppressed the proliferation of A375 cells, induced A375 cells apoptosis, and increased the generation of reactive oxygen species (ROS). In addition, CDK5RAP1 deficiency induced the phosphorylation of $\mathrm{NF}-\kappa \mathrm{B}$ and $\mathrm{Bcl}-2 / \mathrm{Bcl}-\mathrm{xl}$-associated death promoter (Bad). Notably, the phosphorylation of B-cell lymphoma-xl (Bcl-xl) and B-cell lymphoma-2 (Bcl-2) was downregulated by CDK5RAP1 deficiency. Pretreatment with pyrrolidine dithiocarbamate (PDTC), the inhibitor of NF- $\kappa \mathrm{B}$, prevented the decrease in cell proliferation and apoptosis induced by CDK5RAP1 deficiency in A375 cells. However, pretreatment with PDTC did not affect the generation of ROS in A375 cells, indicating that ROS is an upstream target of NF- $\mathrm{B}$ signaling pathway during the apoptosis process. Taken together, CDK5RAP1 deficiency induces cell apoptosis in malignant melanoma A375 cells via the NF- $\kappa \mathrm{B}$ signaling pathway. The results from the present study indicated a potential novel candidate for the treatment of skin cancer.
\end{abstract}

Correspondence to: Professor Yuzhen Li, Department of Dermatology, The Second Affiliated Hospital of Harbin Medical University, 246 Xuefu Road, Harbin, Heilongjiang 150086, P.R. China E-mail: liyuzhen102@126.com

Key words: Cdk5 regulatory subunit-associated protein 1, A375 cells, apoptosis, nuclear factor- $\mathrm{kB}$, reactive oxygen species

\section{Introduction}

Malignant melanoma is a type of malignancy produced by the melanocytes of the skin and other organs (1). Although the occurrence rate of malignant melanoma is low, metastasis occurs is early and the mortality is high (2); therefore, it is necessary to identify a novel treatment strategy for malignant melanoma.

Cdk5 regulatory subunit-associated protein 1 (CDK5RAP1), with homology to the bacterial MiaB protein, is a radical S-Adenosyl methionine (SAM) enzyme $(3,4)$. As presented in Fig. 1, CDK5RAP1 catalyzes the 2-methylthio $\left(\mathrm{ms}^{2}\right)$ modification of mitochondrial transfer $(\mathrm{t}) \mathrm{RNAs}$ at amino acid 37 (5). Deficiencies in the $\mathrm{ms}^{2}$ modification of 2-methylthio-N6-isopentenyladenosine $\left(\mathrm{ms}^{2} \mathrm{i}^{6} \mathrm{~A}\right)$ at amino acid 34 or 37 impair reading frame maintenance in bacteria and cause defective mitochondrial protein synthesis, which leads to a reduction of respiratory activity and increase in ROS (6). The $\mathrm{ms}^{2}$ modifications, which initiate mitochondrial responses, are considered to be the major pathway for apoptosis (7). It has been reported that, CDK5RAP1 deficiency, induces cancer cell apoptosis via the phospho-c-Jun N-terminal kinase (p-JNK) signaling pathway (8).

Apoptosis refers to specific programmed cell death, which is a critical mechanism for cancer therapy. $\mathrm{Ca}^{2+}$ influx, mediated by extracellular signal regulated kinase (ERK1/2) pathway, contributes an important role in early apoptotic cells by activating downstream ROS generation (9). The reactive oxygen species (ROS) mediates cell dysfunction, contributes to the development of cell damage (10), and is responsible for cancer cell apoptosis (11). Nuclear factor- $\kappa \mathrm{B}(\mathrm{NF}-\kappa \mathrm{B})$ pathway is commonly involved in numerous cellular responses (12), and is activated by mitochondrial-generated ROS (13) to induce cell apoptosis (14). The phosphorylation of NF- $\kappa \mathrm{B}$ is associated with the expression of pro-apoptosis B-cell lymphoma-2 (Bcl-2) family, including $\mathrm{Bcl}-2 / \mathrm{Bcl}-\mathrm{xl}$-associated death promoter (Bad), and anti-apoptosis Bcl-2 family, including B-cell lymphoma-xl (Bcl-xl) and Bcl-2 (15). Since targeting mitochondrial modifications is a new field for the treatment of cancer, and apoptosis is linked with $N F-\kappa B$ signaling pathway (16), the aim of the present study was to investigate the relationship between CDK5RAP1 deficiency and NF- $\kappa \mathrm{B}$ 
signaling pathway during the apoptosis process in human malignant melanoma (A375) cells.

In the current study, it was demonstrated that CDK5RAP1 deficiency induces cell apoptosis in malignant melanoma A375 cells via the ROS and NF- $\mathrm{B}$ signaling pathway. This study indicated a unique candidate of anti-skin cancer.

\section{Materials and methods}

Cell culture. The human malignant melanoma A375 cell line (A375-P) was purchased from American Type Culture Collection (Manassas, VA, USA). In accordance with experimental guidelines and ethical approval of Harbin Medical University (Harbin, China), the study was performed in Harbin Medical University. A375 cells were cultured in Dulbecco's modified Eagle's medium (DMEM) supplemented with $10 \%$ fetal bovine serum, $100 \mathrm{U} / \mathrm{ml}$ penicillin and $100 \mathrm{mg} / \mathrm{ml}$ streptomycin (all from Sigma-Aldrich; Merck KGaA, Darmstadt, Germany) with $5 \% \mathrm{CO}_{2}$ at $37^{\circ} \mathrm{C}$ in a humidified incubator (Sanyo Electric Co., Ltd., Tokyo, Japan).

Small interfering RNA (siRNA) transfection. Control siRNA and the CDK5RAP1 siRNA were purchased from Santa Cruz Biotechnology, Inc. (Dallas, TX, USA). A375 cells were seeded at a density of $1 \times 10^{5}$ cells/well onto six-well plates. After cells obtained 60-80\% confluency, siRNAs were transfected into A375 cells according to the manufacturer's protocol. A375 cells were incubated for another $48 \mathrm{~h}$ prior to use in subsequent experiments. The transfection efficiency was analyzed by reverse transcription-quantitative polymerase chain reaction (RT-qPCR), according to the protocol outlined below.

Measurement of cytoplasmic $\mathrm{Ca}^{2+}$ influx. $\mathrm{Ca}^{2+}$ influx was performed as described previously (17). Briefly, A375 cells at a density of $2 \times 10^{6}$ cells $/ \mathrm{ml}$ were loaded with $1 \mu \mathrm{M}$ calcium-sensitive Fura 2-AM in $\mathrm{Ca}^{2+}$-free buffer (Hank's balanced salt solution containing $20 \mathrm{mM}$ HEPES and 1\% bovine serum albumin, $\mathrm{pH}$ 7.4; Sigma-Aldrich; Merck KGaA) at $37^{\circ} \mathrm{C}$ for $30 \mathrm{~min}$. Adenosine triphosphate $(10 \mu \mathrm{M})$ was added to the cell suspension directly. An F-2500 calcium imaging system (Hitachi, Tokyo, Japan) was used to record within FL Solutions. The ratio of fluorescent signals was measured under wavelengths of $340 \mathrm{~nm}, 380 \mathrm{~nm}$ (excitation) and wavelength of $510 \mathrm{~nm}$ (emission). The free Fura 2 and the $\mathrm{Ca}^{2+}$-bound Fura 2 were measured using excitation wavelengths of 380 and $340 \mathrm{~nm}$, respectively. The fluorescent activities of F1 at $340 / 500 \mathrm{~nm}$ and F2 at 380/500 nm, as well as the ratio of F1 to F2 were recorded using the spectrophotometer at $5 \mathrm{sec}$ intervals. The $\mathrm{Ca}^{2+}$ concentration was finally calculated using the formula: $224 x \mathrm{R}$, where 224 is the Kd number.

$R T$ - $q P C R$. Total RNA was extracted from A375 cells by TRIzol ${ }^{\circledR}$ reagent (Life Technologies; Thermo Fisher Scientific, Inc., Waltham, MA, USA) at $48 \mathrm{~h}$ post-transfection of CDK5RAP1 siRNA and control siRNA. Reverse transcription was performed using a Transcriptor First Strand cDNA Synthesis kit (Roche Applied Science, Madison, WI, USA). A total of $200 \mathrm{ng}$ RNA was used for the RT reaction, and $2 \mu \mathrm{l}$ input cDNA from the RT product was used for the qPCR. LightCycler ${ }^{\circledR} 480$ SYBR Green I Master (Roche Diagnostics,
Basel, Switzerland) was used and the qPCR was performed using the ABI 7300 Fast real-time PCR system (Applied Biosystems; Thermo Fisher Scientific, Inc.) with the following primers: CDK5RAP1 forward, 5'-ATGGCTGCCAGATGA ATGTGA-3' and reverse, 5'-CTCTTGGAGGTTACTGGT CCG-3'; 18s forward, 5'-GTA ACCCGTTGA ACCCCA TT-3' and reverse, 5'-CCATCCAATCGGTAGTAGCG-3'. PCR was performed using SYBR Premix Ex Taq II (Takara Bio, Inc., Otsu, Japan). The thermocycling conditions were: Pre-denaturation at $95^{\circ} \mathrm{C}$ for $30 \mathrm{sec}$, denaturation at $95^{\circ} \mathrm{C}$ for $3 \mathrm{sec}$ for 40 cycles, annealing at $60^{\circ} \mathrm{C}$ for $31 \mathrm{sec}$, elongation at $72^{\circ} \mathrm{C}$ for $60 \mathrm{sec}$ and re-elongation at $72^{\circ} \mathrm{C}$ for $5 \mathrm{~min}$. CDK5RAP1 mRNA expression was normalized to $18 \mathrm{~s}$ mRNA expression. $2^{-\Delta \Delta \mathrm{Cq}}$ method was used as the quantification method for the gene of interest, as described previously (18).

MTT assay. The viability of A375 cells, which were transfected with control siRNA and CDK5RAP1 siRNA with or without pyrrolidine dithiocarbamate (PDTC; $100 \mu \mathrm{mol}$ ) treatment, was determined by a colorimetric MTT assay as described previously (19). Cell viability was determined by measuring the absorbance at $550 \mathrm{~nm}$ on a microplate reader. Absorbance at $690 \mathrm{~nm}$ was also measured as the reference wavelength. The measured absorbance under $550 \mathrm{~nm}$ was read as the optical density was used to reflect the number of viable cells.

Nuclear staining with Hoechst 33342 for morphological evaluation. A375 cells were plated in six-well plates at a density of $1 \times 10^{5}$ cells/well and pretreated with or without PDTC (100 $\mu \mathrm{mol})$. Cells were washed with PBS after transfection with CDK5RAP1 siRNA or control siRNA for $48 \mathrm{~h}$. Cells were then fixed in $4 \%$ paraformaldehyde (BIOSS, Beijing, China) for $30 \mathrm{~min}$ at $22^{\circ} \mathrm{C}$ followed by staining with Hoechst 33342 $(20 \mathrm{mg} / \mathrm{ml})$ for $15 \mathrm{~min}$ at room temperature in the dark. Cells were then imaged by using fluorescence microscopy (C1-T-SM; Nikon Corporation, Tokyo, Japan) at a magnification, x100.

Detection of intracellular ROS. Intracellular accumulation of ROS was estimated using the fluorescent dye $\mathrm{H}_{2}$-DCFDA (Thermo Fisher Scientific, Inc.) as described previously (20). The A375 cells were seeded at a density of $1 \times 10^{5}$ cells/well, in a six-well plate, with or without PDTC $(100 \mu \mathrm{mol})$ pretreatment. Following transfection with CDK5RAP1 siRNA or control siRNA for 48 h, A375 cells were washed with serum-free DMEM medium (Sigma-Aldrich; Merck KGaA) and incubated in $\mathrm{H}_{2}$-DCFDA $(5 \mu \mathrm{M})$ for $60 \mathrm{~min}$ at $37^{\circ} \mathrm{C}$. The cells were then examined under a fluorescence microscope (C1-T-SM; Nikon Corporation, Tokyo, Japan). Finally, cells were collected and subjected to fluorescence Spectrophotometer (F-2500; Hitachi, Ltd., Tokyo, Japan) to detect the fluorescence of DCF inside cells (excitation, $488 \mathrm{~nm}$; emission, $521 \mathrm{~nm}$ ).

Western blot analysis. Total proteins were extracted from cells. Cells were lysed by lysis buffer (1 M Tris-HCl, pH 7.4; $1 \mathrm{M} \mathrm{NaCl} ; 20 \%$ Triton X100; 10\% SDS; and 0.5 M EDTA; Sigma-Aldrich; Merck KGaA) and centrifuged at 3,300 x g for $3 \mathrm{~min}$ at $22^{\circ} \mathrm{C}$. Electrophoresis was performed using a vertical slab gel with $12 \%$ polyacrylamide content. A total of $20 \mu \mathrm{g}$ of protein was loaded per gel lane and the transfer of proteins was performed electrophoretically according 
to the method previously described $(21,22)$. Subsequent to treatment with Block Ace ${ }^{\mathrm{TM}}(4 \%)$ for $30 \mathrm{~min}$ at $22^{\circ} \mathrm{C}$, the polyvinylidene fluoride membranes (Thermo Fisher Scientific, Inc.) were probed with rabbit IgG primary antibodies against NF-кB (cat. no., SAB4502609; 1:500; Sigma-Aldrich; Merck KGaA), Bcl-xl (cat. no., SAB4502623; 1:500; Sigma-Aldrich; Merck KGaA) or Bcl-2 (cat. no., SAB4500003; 1:500; Sigma-Aldrich; Merck KGaA) in PBS containing $0.03 \%$ Tween-20 (PBST) for $1 \mathrm{~h}$ at $22^{\circ} \mathrm{C}$. Following three washes with PBST, the second reaction was performed using horseradish peroxidase-conjugated anti-rabbit goat IgG (cat. no., A0545; 20 ng/ml; Sigma-Aldrich; Merck KGaA) secondary antibody for $30 \mathrm{~min}$ at $22^{\circ} \mathrm{C}$. Following three washes with PBST, the membrane was incubated with ECL Plus Western Blotting Detection system $^{\mathrm{TM}}$ (GE Healthcare Life Sciences, Beijing, China) followed by detection. ImageJ (version 1.38e; National Institutes of Health, Bethesda, MD, USA) was used for the quantification of western blots. Histone H1.4 (cat. no., H7665; $2 \mu \mathrm{g} / \mathrm{ml}$; Sigma-Aldrich; Merck KGaA) and $\beta$-actin (cat. no., A5441; 1:5,000; Sigma-Aldrich; Merck KGaA) were used for normalization.

Statistical analysis. Data are expressed as the mean \pm standard deviation. Analyses were performed using SPSS (v.19.0; IBM SPSS, Armonk, NY, USA). Each experiment was repeated at least three times. One-way analysis of variance and Dunnett's test was used and $\mathrm{P}<0.05$ was considered to indicate a statistically significant difference.

\section{Results}

CDK5RAP1 deficiency suppresses cell proliferation of A375 cells. In order to investigate the potential effect of CDK5RAP1, prior to subsequent experiments, human malignant melanoma A375 cells were transfected with siRNA for $48 \mathrm{~h}$. It was confirmed by PCR that CDK5RAP1 knockdown was evident following transfection with CDK5RAP1 siRNA compared with control siRNA in A375 cells (Fig. 2A and B). In addition, the effects of CDK5RAP1 deficiency on the proliferation of A375 cells, was investigated. At $48 \mathrm{~h}$ post-transfection with CDK5RAP1 or control siRNA, the viability of A375 cells was determined by a colorimetric MTT assay. It was demonstrated that CDK5RAP1 deficiency suppressed the proliferation of A375 cells significantly compared with control cells (Fig. 2C a, b and D; C vs. KD; $\mathrm{P}<0.01$ ).

CDK5RAP1 deficiency induces the cell apoptosis in A375 cells. To determine whether CDK5RAP1 knockdown affects the potential apoptosis effect of A375 cells, a series of apoptosis assays were performed. Firstly, $\mathrm{Ca}^{2+}$ influx was measured to estimate the early stage of apoptosis. It was demonstrated that CDK5RAP1 deficiency significantly inhibited $\mathrm{Ca}^{2+}$ influx in A375 cells (Fig. 3A; $\mathrm{P}<0.01$ ). This indicates that A375 cells lost the normal ability of $\mathrm{Ca}^{2+}$ handling, which renders cells to the possibility of apoptosis. Furthermore, apoptosis in cells was examined by nuclear staining with Hoechst 33342 and fluorescence microscopy. CDK5RAP1 deficiency significantly induced A375 cell apoptosis compared with that of control cells (Fig. 3B a, b and C; C vs. KD; P<0.01). To further determine apoptosis in CDK5RAP1 deficient cells, apoptotic
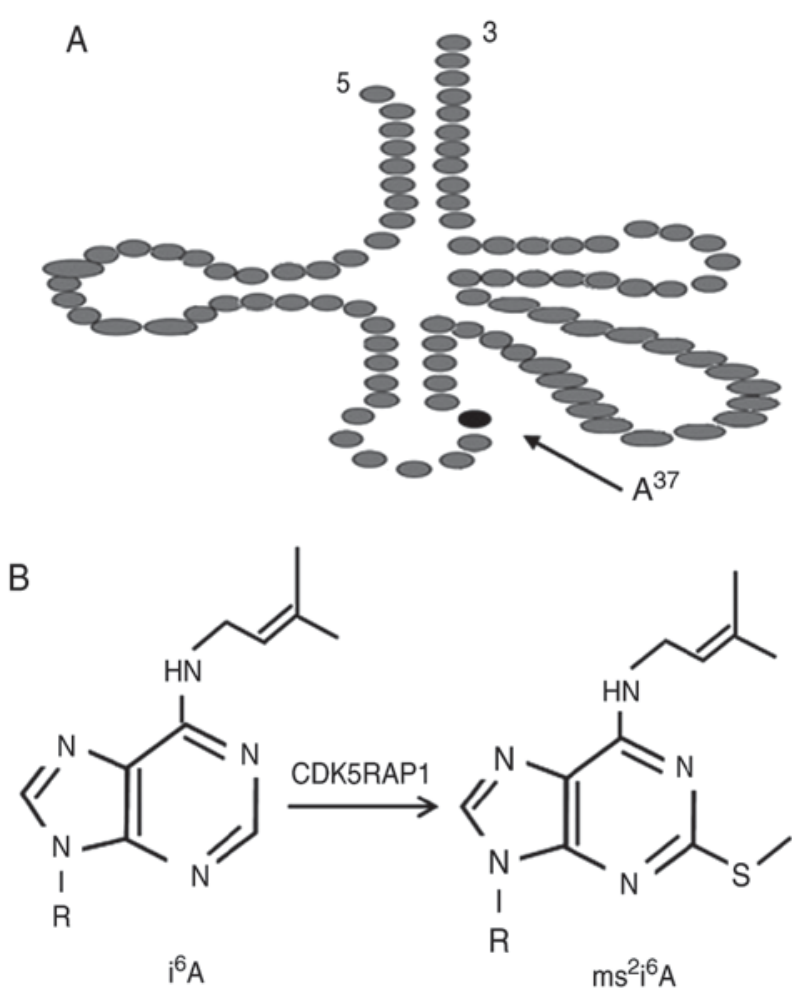

Figure 1. (A) The structure of tRNA. (B) CDK5RAP1 post-synthetically converts the tRNA modification $\mathrm{i}^{6} \mathrm{~A}$ into $\mathrm{ms}^{2} \mathrm{i}^{6} \mathrm{~A}$ at A375 cells. tRNA, transfer RNA. CDK5RAP1, Cdk5 regulatory subunit-associated protein 1. $\mathrm{i}^{6} \mathrm{~A}$, N6-isopentenyladenosine; $\mathrm{ms}^{2} \mathrm{i}^{6} \mathrm{~A}$, 2-methylthio-N6-isopentenyladenosine.

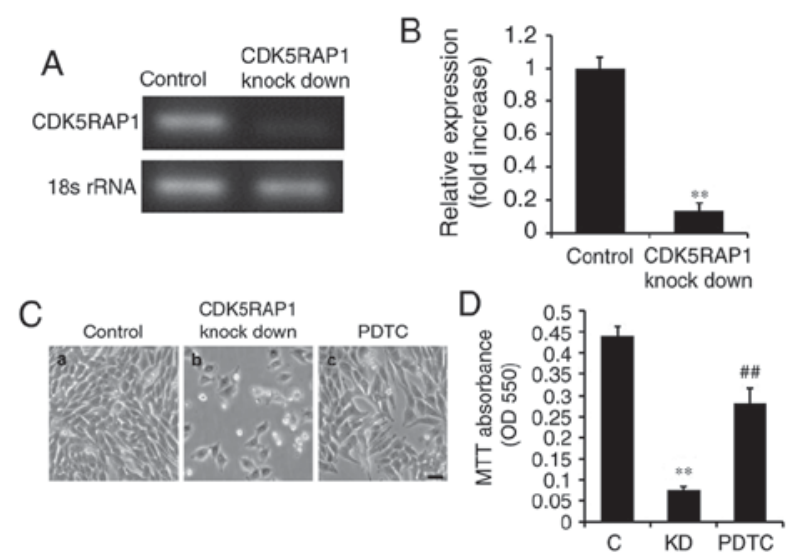

Figure 2. (A) Representative CDK5RAP1 mRNA expression and (B) the band quantification. CDK5RAP1 mRNA expression was confirmed to be knock downed by qPCR in A375 cells transfected with CDK5RAP1 siRNA compared with control siRNA. (C) Representative image of proliferated A375 cells transfected by CDK5RAP1 siRNA with or without the inhibitor of nuclear factor- $\kappa \mathrm{B}$, PDTC pretreatment and control siRNA $(100 \mu \mathrm{mol})(\mathrm{x} 400$ magnification). (D) Relative viability of CDK5RAP1 siRNA-transfected A375 cells with or without PDTC pretreatment, determined by MTT assay. Pretreatment with PDTC prevented the decrease of proliferation induced by CDK5RAP1 deficiency in A375 cells. Data are expressed as the mean \pm SD $(\mathrm{n}=3) .{ }^{* *} \mathrm{P}<0.01, \mathrm{CDK}$ RRAP1 knockdown vs. control; ${ }^{\#} \mathrm{P}<0.01$, PDTC vs. CDK5RAP1 knockdown. Scale bar $=20 \mu \mathrm{m}$. C, control; KD, CDK5RAP1 knockdown; siRNA, small interfering RNA; CDK5RAP1, Cdk5 regulatory subunit-associated protein 1; PDTC, pyrrolidine dithiocarbamate.

markers of the $\mathrm{Bad} / \mathrm{Bcl}$ signaling pathway were detected by western blot analysis. It was demonstrated that Bad protein was upregulated and its inhibitors, Bcl-xl and Bcl-2, were 

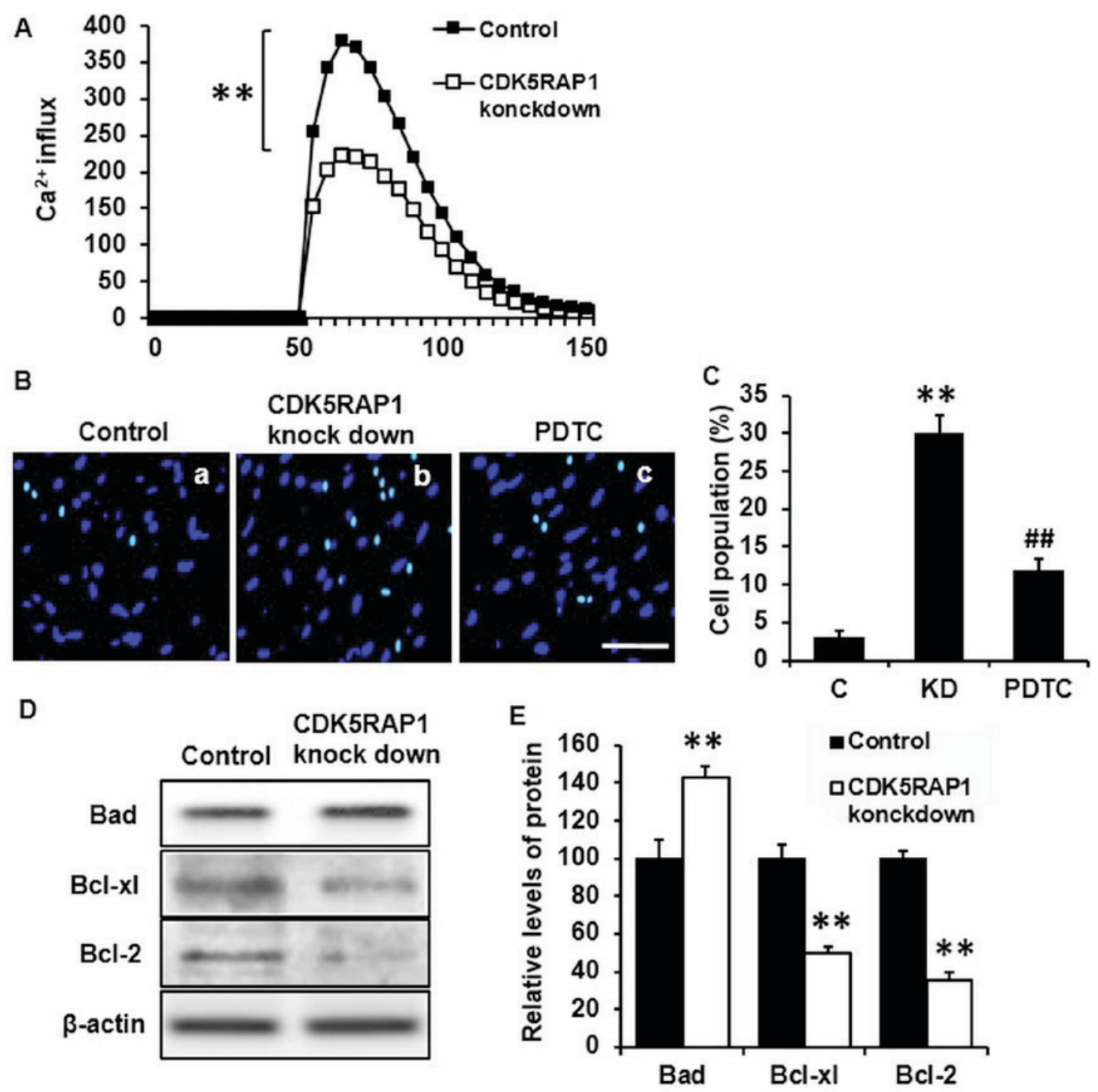

Figure 3. (A) Representative recording image of $\mathrm{Ca}^{2+}$ influx in A375 cells transfected by CDK5RAP1 siRNA and control siRNA. CDK5RAP1 deficiency significantly inhibited the $\mathrm{Ca}^{2+}$ influx in A375 cells. (B) Representative image of apoptotic A3735 cells transfected by CDK5RAP1 siRNA with or without PDTC pretreatment ( $\mathrm{x} 400$ magnification; Scale bar $=20 \mu \mathrm{m}$.) and $(\mathrm{C})$ quantification of apoptotic cells percentage. Pretreatment with PDTC prevented the apoptosis induced by CDK5RAP1 deficiency in A375 cells significantly. (D) Representative western blot of phosphorylation of Bad, Bcl-xl and Bcl-2 in A375 cells transfected by CDK5RAP1 siRNA and (E) quantification. CDK5RAP1 deficiency significantly upregulated the phosphorylation of Bad, and downregulated the phosphorylation of Bcl-xl and Bcl-2. $\beta$-actin was used as the normalization respectively. Data are expressed as the mean \pm SD $(n=3)$. ** $\mathrm{P}<0.01, \mathrm{CDK} 5 \mathrm{RAP1}$ knockdown vs. control; \# ${ }^{\#}<0.01$, PDTC vs. CDK5RAP1 knockdown. C, control; KD, CDK5RAP1 knockdown; siRNA, small interfering RNA; CDK5RAP1, Cdk5 regulatory subunit-associated protein 1; Bad, Bcl-xl-associated death promoter; Bcl-xl, B-cell lymphoma-xl; Bcl-2, B-cell lymphoma; PDTC, pyrrolidine dithiocarbamate.

downregulated in CDK5RAP1 deficient cells (Fig. 3D and E). Taken together, these results indicated a consistent conclusion that CDK5RAP1 deficiency induced apoptosis of A375 cells.

CDK5RAP1 deficiency induces ROS generation in A375 cells. To explore the mechanism of this aforementioned apoptotic effect in A375 cells, ROS was further investigated, as ROS is considered to induce, and accompanies, cancer cell apoptosis. Thus, the intracellular generation of ROS was detected using the fluorescent dye $\mathrm{H}_{2}$-DCFDA. CDK5RAP1 deficiency induced ROS generation and accumulation in A375 cells significantly (Fig. 4A a, b and B; C vs. KD; P<0.01).

CDK5RAP1 deficiency upregulates the phosphorylation of $N F-\kappa B$. To further study the mechanisms of this beneficial effect of CDK5RAP1 deficiency, western blot analysis for $\mathrm{NF}-\kappa \mathrm{B}$ was performed. It was revealed that the phosphorylation of NF- $\mathrm{NB}$ in A375 cells was significantly upregulated in CDK5RAP1-deficient cells compared with the control cells. Histone 1.4 and $\beta$-actin were used for normalization (Fig. 4C and $\mathrm{D} ; \mathrm{P}<0.01)$.

Pretreatment with PDTC prevents the decrease in proliferation and increases apoptosis induced by CDK5RAP1 deficiency in A375 cells. As it was demonstrated that CDK5RAP1 deficiency induced $\mathrm{NF}-\kappa \mathrm{B}$ expression, it was imperative to further explore if the NF- $\kappa$ B pathway was sufficient to explain the effects of CDK5RAP1 deficiency. To determine this, NF- $\kappa \mathrm{B}$ was inhibited using PDTC (100 $\mu \mathrm{mol})$ prior to transfection with CDK5RAP1 siRNA. Firstly, the viability of CDK5RAP1-deficient A375 
A

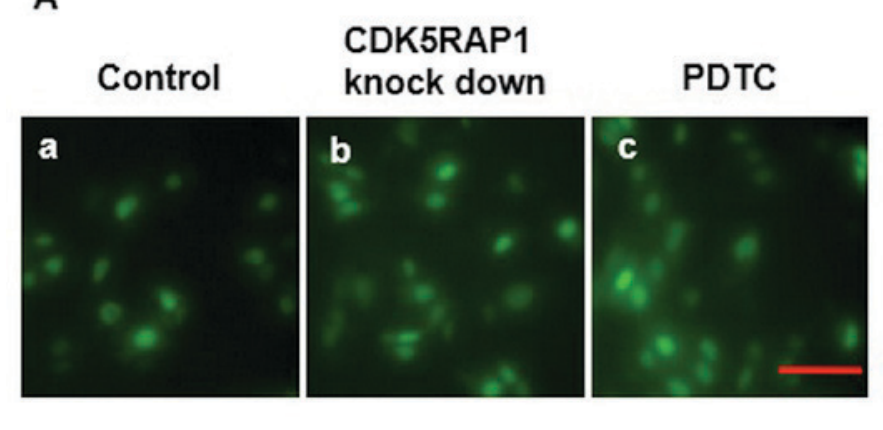

C

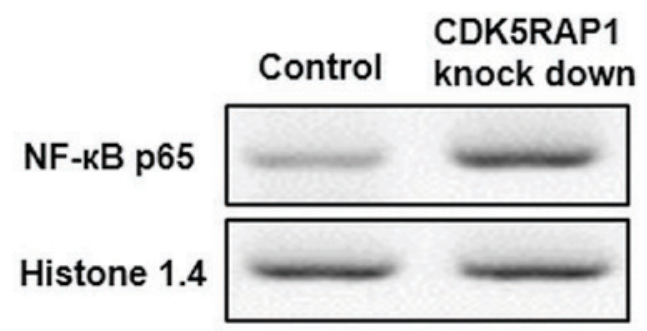

B


Figure 4. (A) Representative image of ROS accumulation in A375 cells transfected with CDK5RAP1 siRNA with or without PDTC pretreatment (x400 magnification; Scale bar size $=20 \mu \mathrm{m}$ ) and $(\mathrm{B})$ quantification of fluorescence intensity percentage. Pretreatment with PDTC did not suppress the ROS generation induced by CDK5RAP1 deficiency in A375 cells. (C) Representative western blot of phosphorylation of NF- $\kappa$ B (p65) in A375 cells transfected by CDK5RAP1 siRNA and (D) quantification. NF- $\mathrm{BB}$ p65 was significantly induced in the CDK5RAP1 deficiency cells. ${ }^{* *} \mathrm{P}<0.01, \mathrm{CDK} 5 \mathrm{RAP} 1 \mathrm{knockdown}$ vs. control. C, control; KD, CDK5RAP1 knockdown; NF- $\mathrm{B}$, nuclear factor- $\kappa \mathrm{B}$; siRNA, small interfering RNA; CDK5RAP1, Cdk5 regulatory subunit-associated protein 1; PDTC, pyrrolidine dithiocarbamate.

cells pretreated with PDTC was measured using a colorimetric MTT assay. It was revealed that NF- $\mathrm{B}$ inhibition, by PDTC, prevented the decrease in proliferation induced by CDK5RAP1 deficiency in A375 cells (Fig. 2C b, c and D; KD vs. PDTC; $\mathrm{P}<0.01)$. In addition, $\mathrm{NF}-\kappa \mathrm{B}$ inhibition, by PDTC, prevented the apoptosis induced by CDK5RAP1 deficiency in A375 cells (Fig. 3B b, c and C; KD a vs. PDT; P<0.01). However, notably, CDK5RAP1 deficiency induced-ROS was not significantly inhibited by pretreatment with PDTC (Fig. 4A b, c and B; KD vs. PDTC). Taken together, the results from the present study indicated that NF- $\kappa \mathrm{B}$ may contribute in the process of CDK5RAP1 deficiency-induced apoptosis, and in contrast, it is possible that $\mathrm{NF}-\kappa \mathrm{B}$ was not upstream of ROS production.

\section{Discussion}

To the best of our knowledge, the present study is the first to report that CDK5RAP1 deficiency promoted apoptosis in the malignant melanoma cell line, A375. This favorable effect on A375 cells was induced by ROS generation and NF- $\mathrm{NB}$ activation, which was necessary to explain the pathway of CDK5RAP1 deficiency induced A375 cells apoptosis.

CDK5RAP1, with homology to the bacterial MiaB protein, is a radical SAM enzyme $(3,4)$. CDK5RAP1 catalyzes 2-methylthio $\left(\mathrm{ms}^{2}\right)$ modification of mitochondrial tRNAs (i ${ }^{6} \mathrm{~A}$ into $\mathrm{ms}^{2} \mathrm{i}^{6} \mathrm{~A}$ ) at $\mathrm{A} 37$ (5). The $\mathrm{ms}^{2}$ modifications optimize mitochondrial translation $(23,24)$. CDK5RAP1 established the biochemical association between the enzymatic modification and cell apoptosis (6). Deficiency in $\mathrm{ms}^{2}$ modification has been demonstrated to markedly impaired mitochondrial protein synthesis, which results in a number of diseases, including vitiligo and type 2 diabetes $(6,25)$. It has been suggested that deficiency in $\mathrm{ms}^{2}$ modification induced by CDK5RAP1 deficiency induces cancer cell apoptosis via the p-JNK signaling pathway (8).

Apoptosis is an essential mechanism. Numerous chemotherapeutic agents inhibit tumor growth through suppressing apoptosis of cancer cells (26). $\mathrm{Ca}^{2+}$ influx serves an important function and is associated with the phosphatide-conjugated protein, Annexin V, which has presented a high binding affinity to phosphatidylserine of apoptotic cells in early stages (9). Apoptosis is also considered to be associated with mitochondria-initiated responses. As CDK5RAP1 deficiency-induced deficiency in $\mathrm{ms}^{2}$ modification is closely associated with cancer cell apoptosis, CDK5RAP1 deficiency may have a beneficial effect on inhibiting cancer process. According to these theories, the present study demonstrated that CDK5RAP1 deficiency inhibited the $\mathrm{Ca}^{2+}$ influx in human malignant melanoma A375 cells, and suppressed the proliferation and induced apoptosis of A375 cells. 

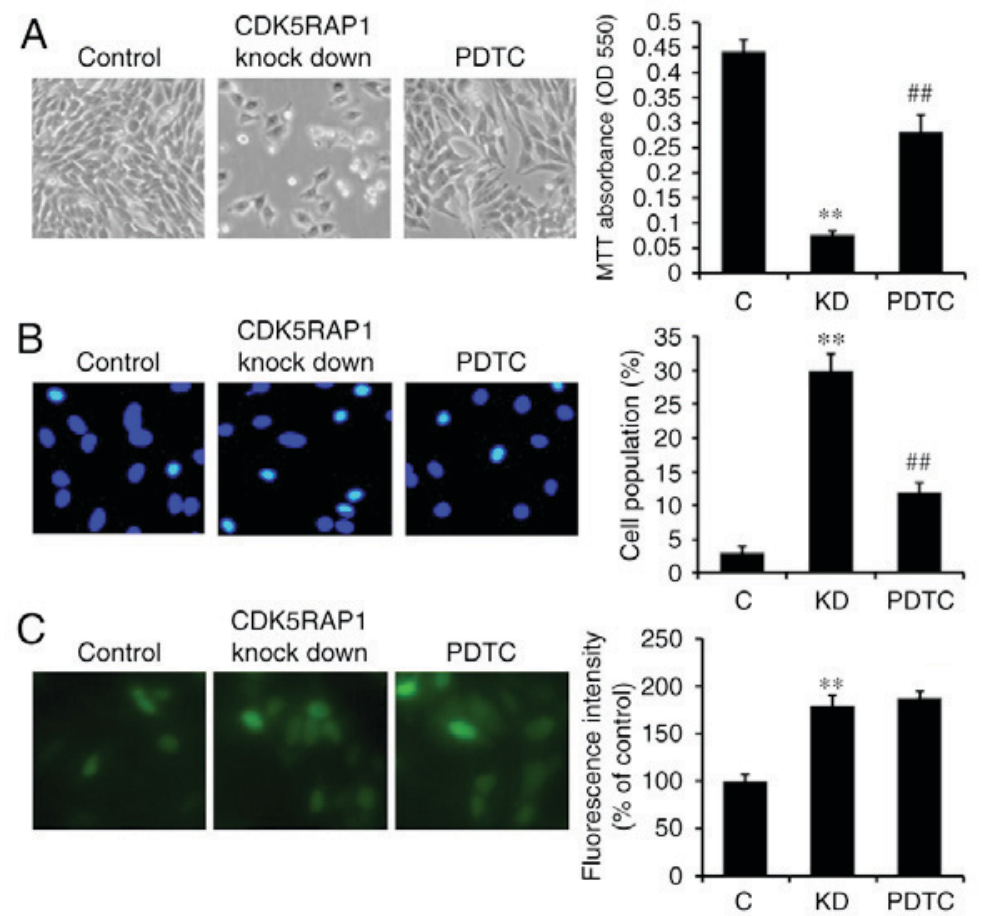

Figure 5. (A) Representative image of proliferated A375 cells transfected by CDK5RAP1 siRNA and control siRNA with or without PDTC (100 $\mu$ mol), the inhibitor of NF- $\mathrm{KB}$, pretreatment (left). Relative viability of CDK5RAP1 siRNA transfected A375 cells with or without PDTC pretreatment, determined by MTT assay (right). Pretreatment with PDTC prevented the decrease of proliferation induced by CDK5RAP1 deficiency in A375 cells. (B) Representative image of apoptotic A3735 cells transfected by CDK5RAP1 siRNA with or without PDTC (left) and the quantification of apoptotic cells percentage (right). Pretreatment with PDTC prevented the apoptosis induced by CDK5RAP1 deficiency in A375 cells significantly. (C) Representative image of ROS accumulation in A375 cells transfected with CDK5RAP1 siRNA with or without PDTC pretreatment (left) and quantification of fluorescence intensity percentage (right). Pretreatment with PDTC did not suppress ROS generation induced by CDK5RAP1 deficiency in A375 cells. Data are expressed as the mean \pm SD $(\mathrm{n}=3) .{ }^{* *} \mathrm{P}<0.01, \mathrm{CDK} 5 \mathrm{R} A P 1$ knockdown vs. control; ${ }^{\# \#} \mathrm{P}<0.01$, PDTC vs. CDK5RAP1 knockdown ( 4400 magnification). C, control; KD, CDK5RAP1 knockdown; siRNA, small interfering RNA; CDK5RAP1, Cdk5 regulatory subunit-associated protein 1; ROS, reactive oxygen species; OD, optical density; PDTC, pyrrolidine dithiocarbamate.

ROS, the normal cellular oxidative process byproduct, has been reported in regulating apoptotic initiation signaling and is associated with several oncogenic pathways (27). Thus, the beneficial role to suppress ROS generation and accumulation is evident. There is compelling evidence that only ROS, which overcomes cellular antioxidant defenses, may trigger apoptosis and facilitate cancer cells more sensitive to ROS compared with normal cells (10). Basal ROS levels elevate oncogenic transformation significantly, thus apoptotic programming in cancer cells is easily triggered by further acute increases in ROS (11). Redundant ROS initiates cytotoxicity, which is also considered to be an important mechanism in the anticancer mechanism (28). Consistently, the results of the current study demonstrated that CDK5RAP1 deficiency significantly promoted ROS generation and accumulation in A375 cells, suggesting that apoptosis induced by CDK5RAP1 deficiency in A375 cells is promoted through increased ROS production.

ROS generated by mitochondria has been demonstrated to activate NF- $\kappa \mathrm{B}$ (13). NF- $\kappa \mathrm{B}$ activation through the ROS-dependent pathway induces cell apoptosis (14). $\mathrm{NF}-\kappa \mathrm{B}$, a transcription factor, usually exists as an inactive form through binding to its inhibitory protein, I $\mathrm{K}$ in the cytoplasm (12). The nuclear NF- $\mathrm{B}$ family is well known in regulating inflammatory responses, which are also important for cancer cell apoptosis (29). It has also been reported that apoptosis is associated with NF- $\mathrm{kB}(16)$ and

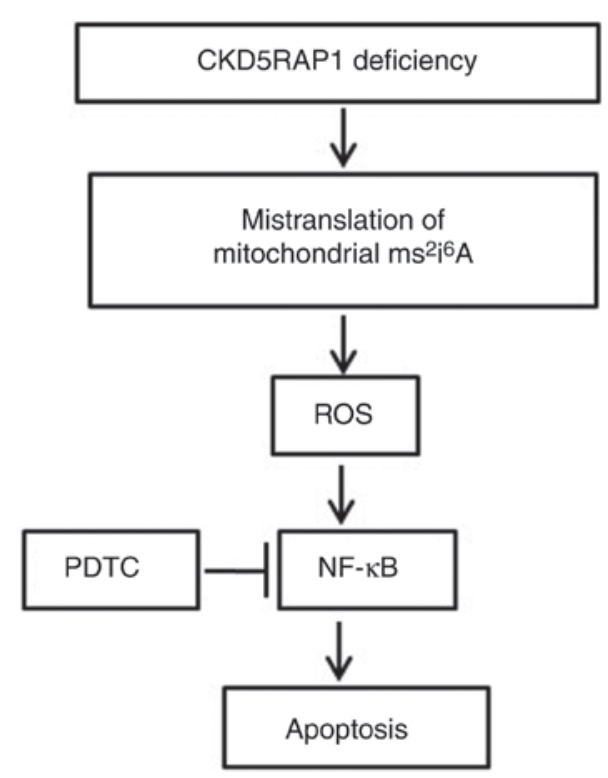

Figure 6. Mechanism of the apoptosis process in A375 cells. CDK5RAP1 deficiency induces the mistranslation of mitochondrial $\mathrm{ms}^{2} \mathrm{i}^{6} \mathrm{~A}$, induced the generation of ROS and the phosphorylation of NF- $\mathrm{kB}$, resulting in apoptosis of A375 cells. PDTC, the inhibitor of NF-אB, prevents the decrease of proliferation and the apoptosis induced by CDK5RAP1 deficiency in A375 cells, but does not affect the generation of ROS in A375 cells, indicating that the ROS pathway is upstream of NF- $\mathrm{KB}$ signaling pathway during the apoptosis process. ROS, reactive oxygen species; CDK5RAP1, Cdk5 regulatory subunit-associ-

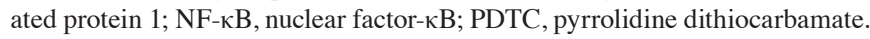


the activation of NF- $\mathrm{B}$ plays pivotal roles in apoptosis (30). It is well documented that NF- $\mathrm{NB}$ has bidirectional modulatory effects on cell apoptosis $(31,32)$. The phosphorylation of NF- $\mathrm{BB}$ is associated with the pro-apoptosis Bcl-2 family, including Bad, and anti-apoptosis Bcl-2 family, including Bcl-xl and Bcl-2 (15). As demonstrated in the present study, CDK5RAP1 deficiency significantly induced the phosphorylation of $N F-\kappa B$ and Bad in human malignant melanoma A375 cells, confirming that the $\mathrm{NF}-\kappa \mathrm{B}$ signaling pathway was targeted by mitochondria modification. Notably, the phosphorylation of Bcl-xl and Bcl-2 was downregulated by CDK5RAP1 deficiency.

To better understand the signaling pathway in the apoptosis process, PDTC, an inhibitor of NF- $\kappa \mathrm{B}$, was used. Pretreatment with PDTC prevented the decrease in proliferation and apoptosis induced by CDK5RAP1 deficiency in A375 cells. However, pretreatment with PDTC did not affect the generation of ROS in A375 cells, indicating that the ROS signaling pathway is upstream of the NF- $\kappa \mathrm{B}$ signaling pathway during the apoptosis process.

CDK5RAP1 is associated with mitochondrial modification, thus CDK5RAP1 deficiency leads to mitochondrial dysfunction. However, as a limitation, CDK5RAP1 does not exist only in cancer cells, but also in all normal cells. This limits the use of CDK5RAP1 deficiency for therapy. In order for CDK5RAP1 deficiency to be used in gene therapy, it is important to develop a means of specifically targeting CDK5RAP1 in cancer cells.

To the best of our knowledge, this is the first study to demonstrate that CDK5RAP1 deficiency induces apoptosis in human malignant melanoma A375 cells via the ROS/NF- $\kappa \mathrm{B}$ signaling pathway. Although the present data provides evidence that cancer progression was remarkably inhibited in CDK5RAP1 deficient cells, practical application still needs to be further investigated. The potential effects of CDK5RAP1 deficiency in cancer cells is expected to provide important insight for developing a novel clinical cancer therapy.

\section{Competing interests}

The authors declare that they have no competing interests.

\section{References}

1. Hatanaka M, Higashi Y, Kawai K, Su J, Zeng W, Chen X and Kanekura T: CD147-targeted siRNA in A375 malignant melanoma cells induces the phosphorylation of EGFR and downregulates cdc25C and MEK phosphorylation. Oncol Lett 11: 2424-2428, 2016.

2. Ostheimer C, Bormann C, Fiedler E, Marsch W and Vordermark D: Malignant melanoma brain metastases: Treatment results and prognostic factors-a single-center retrospective study. Int J Oncol 46: 2439-2448, 2015.

3. Reiter V, Matschkal DM, Wagner M, Globisch D, Kneuttinger AC, Müller M and Carell T: The CDK5 repressor CDK5RAP1 is a methylthiotransferase acting on nuclear and mitochondrial RNA Nucleic Acids Res 40: 6235-6240, 2012.

4. Zou X, Ji C, Jin F, Liu J, Wu M, Zheng H, Wang Y, Li X, $\mathrm{Xu} \mathrm{J}, \mathrm{Gu} \mathrm{S}$, et al: Cloning, characterization and expression of CDK5RAP1_v3 and CDK5RAP1_v4, two novel splice variants of human CDK5RAP1. Genes Genet Syst 79: 177-182, 2004.

5. Pierrel F, Douki T, Fontecave M and Atta M: MiaB protein is a bifunctional radical-S-adenosylmethionine enzyme involved in thiolation and methylation of tRNA. J Biol Chem 279: 47555-47563, 2004.
6. Wei FY, Zhou B, Suzuki T, Miyata K, Ujihara Y, Horiguchi H, Takahashi N, Xie P, Michiue H, Fujimura A, et al: Cdk5rapl-mediated 2-methylthio modification of mitochondrial tRNAs governs protein translation and contributes to myopathy in mice and humans. Cell Metab 21: 428-442, 2015.

7. Wang H, Wei L, Li C, Zhou J and Li Z: CDK5RAP1 deficiency induces cell cycle arrest and apoptosis in human breast cancer cell line by the ROS/JNK signaling pathway. Oncol Rep 33: 1089-1096, 2015.

8. Brody JG, Rudel RA, Michels KB, Moysich KB, Bernstein L, Attfield KR and Gray S: Environmental pollutants, diet, physical activity, body size, and breast cancer: Where do we stand in research to identify opportunities for prevention? Cancer 109 (12 Suppl): S2627-S2634, 2007.

9. Li X, Zhao H, Wang Q, Liang H and Jiang X: Fucoidan protects ARPE-19 cells from oxidative stress via normalization of reactive oxygen species generation through the $\mathrm{Ca}^{2+}$-dependent ERK signaling pathway. Mol Med Rep 11: 3746-3752, 2015.

10. Myatt SS, Brosens JJ and Lam EW: Sense and sensitivity: FOXO and ROS in cancer development and treatment. Antioxid Redox Signal 14: 675-687, 2011.

11. Wang H, Zhang T, Sun W, Wang Z, Zuo D, Zhou Z, Li S, Xu J, Yin F, Hua $Y$ and Cai Z: Erianin induces G2/M-phase arrest, apoptosis, and autophagy via the ROS/JNK signaling pathway in human osteosarcoma cells in vitro and in vivo. Cell Death Dis 7: e2247, 2016.

12. Kurihara $Y$ and Furue M: Interferon- $\gamma$ enhances phorbol myristate acetate-induced cell attachment and tumor necrosis factor production via the NF- $\kappa$ B pathway in THP-1 human monocytic cells. Mol Med Rep 7: 1739-1744, 2013.

13. Li F, Ambrosini G, Chu EY, Plescia J, Tognin S, Marchisio PC and Altieri DC: Control of apoptosis and mitotic spindle checkpoint by survivin. Nature 396: 580-584, 1998.

14. Chandel NS, Trzyna WC, McClintock DS and Schumacker PT: Role of oxidants in NF- $\kappa$ B activation and TNF-alpha gene transcription induced by hypoxia and endotoxin. J Immunol 165 : 1013-1021, 2000.

15. Turillazzi E, Neri M, Cerretani D, Cantatore S, Frati P, Moltoni L, Busardò FP, Pomara C, Riezzo I and Fineschi V: Lipid peroxidation and apoptotic response in rat brain areas induced by long-term administration of nandrolone: The mutual crosstalk between ROS and NF-kB. J Cell Mol Med 20: 601-612, 2016.

16. Lou L, Zhou J, Liu Y, Wei YI, Zhao J, Deng J, Dong B, Zhu L, Wu A, Yang Y and Chai L: Chlorogenic acid induces apoptosis to inhibit inflammatory proliferation of IL-6-induced fibroblast-like synoviocytes through modulating the activation of JAK/STAT and NF- $\mathrm{KB}$ signaling pathways. Exp Ther Med 11: 2054-2060, 2016.

17. Nishiura H, Tokita K, Li Y, Harada K, Woodruff TM, Taylor SM, Nsiama TK, Nishino $\mathrm{N}$ and Yamamoto T: The role of the ribosomal protein S19 C-terminus in Gi protein-dependent alternative activation of p38 MAP kinase via the C5a receptor in HMC-1 cells. Apoptosis 15: 966-981, 2010.

18. Livak KJ and Schmittgen TD: Analysis of relative gene expression data using real-time quantitative PCR and the 2(-Delta Delta C(T)) method. Methods 25: 402-408, 2001.

19. Yang L, Shu T, Liang Y, Gu W, Wang C, Song X, Fan C and Wang W: GDC-0152 attenuates the malignant progression of osteosarcoma promoted by ANGPTL2 via PI3K/AKT but not p38MAPK signaling pathway. Int J Oncol 46: 1651-1658, 2015.

20. Li X, Zhao H, Wang Q, Liang H and Jiang X: Fucoidan protects ARPE-19 cells from oxidative stress via normalization of reactive oxygen species generation through the $\mathrm{Ca}^{2+}$-dependent ERK signaling pathway. Mol Med Rep 11: 3746-3752, 2015.

21. Xu Y, Tian Z and Xie P: Targeting complement anaphylatoxin C5a receptor in hyperoxic lung injury in mice. Mol Med Rep 10: 1786-1792, 2014

22. Jiang X, Yu J, Ma Z, Zhang $\mathrm{H}$ and Xie F: Effects of fucoidan on insulin stimulation and pancreatic protection via the cAMP signaling pathway in vivo and in vitro. Mol Med Rep 12: 4501-4507, 2015.

23. Moncini S, Bevilacqua A, Venturin M, Fallini C, Ratti A, Nicolin A and Riva P: The 3'untranslated region of human Cyclin-Dependent kinase 5 regulatory subunit 1 contains regulatory elements affecting transcript stability. BMC Mol Biol 8: 111, 2007.

24. Jenner LB, Demeshkina N, Yusupova G and Yusupov M: Structural aspects of messenger RNA reading frame maintenance by the ribosome. Nat Struct Mol Biol 17: 555-560, 2010. 
25. Shin MK, Uhm YK, Lee JH, Kim SK, Chung JH and Lee MH: Association between CDK5RAP1 polymorphisms and susceptibility to vitiligo in the Korean population. Eur J Dermatol 22: 495-499, 2012.

26. Matsumoto T, Jimi S, Migita K, Takamatsu Y and Hara S: Inhibition of glucose transporter 1 induces apoptosis and sensitizes multiple myeloma cells to conventional chemotherapeutic agents. Leuk Res 41: 103-110, 2016.

27. Dewaele M, Maes H and Agostinis P: ROS-mediated mechanisms of autophagy stimulation and their relevance in cancer therapy. Autophagy 6: 838-854, 2010.

28. Zou P, Zhang J, Xia Y, Kanchana K, Guo G, Chen W, Huang Y, Wang Z, Yang S and Liang G: ROS generation mediates the anti-cancer effects of WZ35 via activating JNK and ER stress apoptotic pathways in gastric cancer. Oncotarget 6: 5860-5876, 2015.

29. Padua $\mathrm{MB}$ and Hansen PJ: Changes in expression of cell-cycle-related genes in PC-3 prostate cancer cells caused by ovine uterine serpin. J Cell Biochem 107: 1182-1188, 2009.
30. Chu $\mathrm{H}, \mathrm{Yu} \mathrm{H}$, Ren D, Zhu K and Huang H: Plumbagin exerts protective effects in nucleus pulposus cells by attenuating hydrogen peroxide-induced oxidative stress, inflammation and apoptosis through NF- $\kappa \mathrm{B}$ and Nrf-2. Int J Mol Med 37: 1669-1676, 2016.

31. Baldwin AS: Control of oncogenesis and cancer therapy resistance by the transcription factor NF-kappaB. J Clin Invest 107: 241-246, 2001

32. Yang G, Xiao X, Rosen DG, Cheng X, Wu X, Chang B, Liu G, Xue F, Mercado-Uribe I, Chiao P, et al: The biphasic role of NF-kappaB in progression and chemoresistance of ovarian cancer. Clin Cancer Res 17: 2181-2194, 2011.

(i) (2) This work is licensed under a Creative Commons C. Attribution-NonCommercial-NoDerivatives 4.0 International (CC BY-NC-ND 4.0) License. 